\title{
Low Diagnostic Value of Synovial Aspiration Culture Prior to Reimplantation in Periprosthetic Joint Infection
}

\author{
CHRISTIAN MACKE, STEFAN LENHOF, TILMAN GRAULICH, MARCUS ÖRGEL, \\ TAREK OMAR-PACHA, TIMO STÜBIG, CHRISTIAN KRETTEK and MOHAMED OMAR \\ Trauma Department, Hannover Medical School (MHH), Hannover, Germany
}

\begin{abstract}
Background/Aim: We aimed to determine the diagnostic value of the synovial aspiration culture prior to reimplantation in two- (or more) stage exchange of periprosthetic joint infection. Patients and Methods: This was a retrospective study, spanning over ten years including all synovial cultures of patients with two-(or more) stage exchange due to periprosthetic joint infection. Results: A total of 183 patients were included, mean age was 66.6 years (range $=12.8-93.4$ years). Overall sensitivity of synovial aspiration cultures before reimplantation was 56.6\%, specificity $84.6 \%$, negative predictive value (NPV) 63.8\%, positive predictive value (PPV) $80.2 \%$, area under the curve (AUC) $70.6 \%$. Sensitivity of the knee in comparison to the hip culture was significantly higher, as well as the NPV and the AUC ( $p=0.038)$. In case of complete removal of prosthesis, the sensitivity and AUC were significantly reduced, whereas the specificity was comparable with prosthesis in situ, partial removal or complete removal. Conclusion: Due to the low sensitivity, obtaining several synovial cultures in the prosthesis-free interval to exclude persistence of infection, does not seem reasonable.
\end{abstract}

Together with aseptic loosening periprosthetic joint infection (PJI) is the second most common reason for implant failure in arthroplasty (1). The incidence ranges from approximately $1 \%$ in primary arthroplasty and up to $6 \%$ in case of revision arthroplasty (2-5) and the treatment of PJI can be challenging, long and leads to high socio-economic costs (1). In order to perform a purposeful therapy, a sufficient diagnostic work-up

This article is freely accessible online.

Correspondence to: Christian Macke, Hannover Medical School (MHH), Carl-Neuberg-Strasse 1, 30625 Hannover, Germany. Tel: +49 17615322533, Fax: +49 5115328805, e-mail: macke.christian@mhhannover.de

Key Words: Arthroplasty, periprosthetic joint infection, synovial culture, diagnostic value, rule-out test, implant removal. with identification of the pathogenic agent is indispensable (6). The synovial culture is one of the essential components in the diagnostic field of PJI $(7,8)$. It is a fast, minimallyinvasive and low-cost method and allows not only the diagnosis of PJI but also the identification of the pathogenic agent and its resistogram $(3,7,8)$. Although multiple studies address the diagnostic value of the synovial aspiration cultures for primary diagnostic of PJI, data regarding the value during the treatment process is inconsistent. Questions regarding the value of aspiration culture before reimplantation in two or more stage exchanges, the number of aspirations and the influence of antibiotic treatment on diagnostic performance remain unanswered.

The aim of this study was to determine the diagnostic value of the synovial aspiration culture in the process of the complex PJI treatment, with the main focus on hip and knee arthroplasty and the prosthesis-free interval. Therefore, we conducted this retrospective study over ten years on a LevelI university centre on patients treated for periprosthetic infections of the hip and knee. We hypothesize that synovial aspiration culture prior to reimplantation is not suitable to rule out a persistent infection.

\section{Patients and Methods}

Ethical approval. Approval for this study was obtained from our Institution's Ethical Committee as a waiver. Due to the retrospective nature of the study our hospital's general consent for utilization of anonymized research data was eligible.

Study design. Retrospective identification of all cases between January 2005 and December 2015 with the diagnosis "infection or inflammatory reaction due to internal joint prosthesis" (T84.5 in ICD-10 classification) in a level I trauma centre and at least one surgical procedure related to this diagnosis. Among these, all patients with periprosthetic joint infection were selected according the criteria defined below.

Inclusion was done according to the flow chart (Figure 1). A case was defined as a complete treatment period of the infectious arthroplasty from first diagnosis until infection eradication was achieved. As soon as recurrence of infection occurred, a new case was created. Thus, one patient could account for more than one case. 


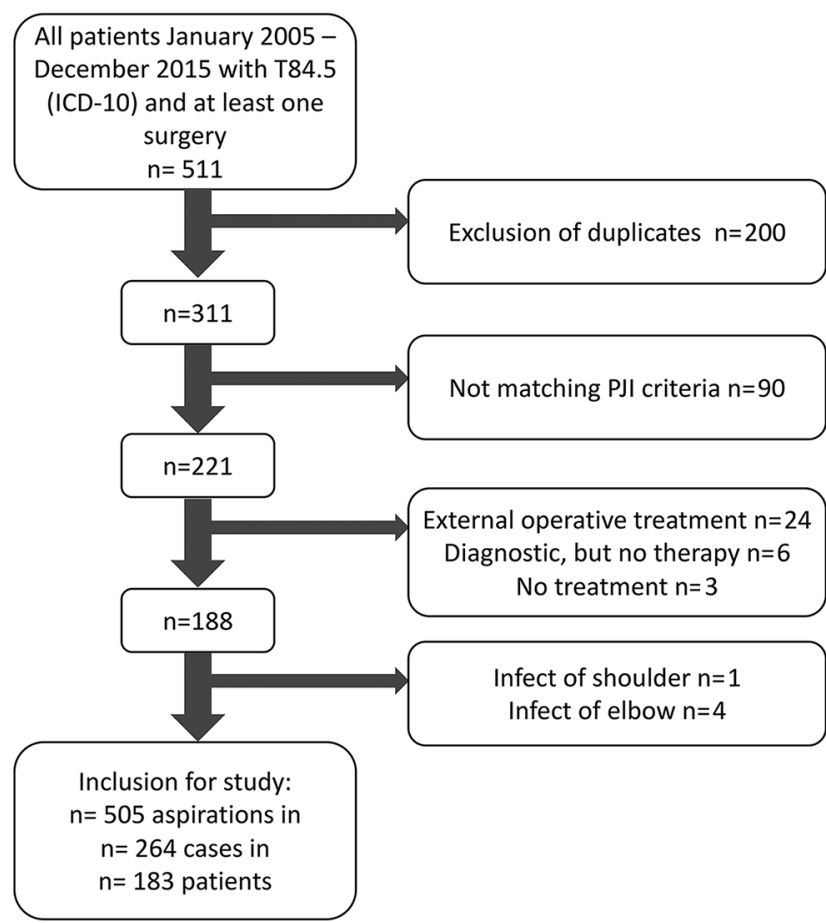

Figure 1. Flowchart for patient inclusion and exclusion. After exclusion criteria 183 patients could be included, resulting in 264 cases, as some patients had recurrent infection and could be included for more than one case.

Periprosthetic Joint Infection (PJI). PJI was defined according to a modified consensus classification of the Musculoskeletal Society when one of the following parameters was fulfilled (7):

- Sinus tract or open wound in communication with the prosthesis;

- Intraoperative purulence;

- At least two positive tested intraoperative cultures or one positive highly pathogenic agent;

- Histological detection of a periprosthetic membrane type II or III in Krenn and Morawietz classification $(9,10)$.

Definition of prosthetic-infection timing. Early (3 Month), delayed (3-24 month) and late (>24 month) infect were assessed according to Zimmerli et al. $(5,11)$.

Collection of data. General patient data, medical history and blood levels of infectious parameters (serum C-reactive protein (CRP) and peripheral blood leukocytes) were collected from the patients' records. As there are multiple synovial aspirations within one case, it was necessary to subdivide every case in defined cycles as shown in Figure 2. The first cycle was from beginning of symptoms to the end of the first operation. Right after that, the next cycle starts and ends with the next operation. The last cycle ends with the termination of the case including the postoperative phase.

Data analysis and statistics. For evaluation of the diagnostic value of the synovial culture every cycle was rated independently for presence of infection according to Renner et al. (7). A PJI was assumed if at least one of the above-mentioned criteria was positive.

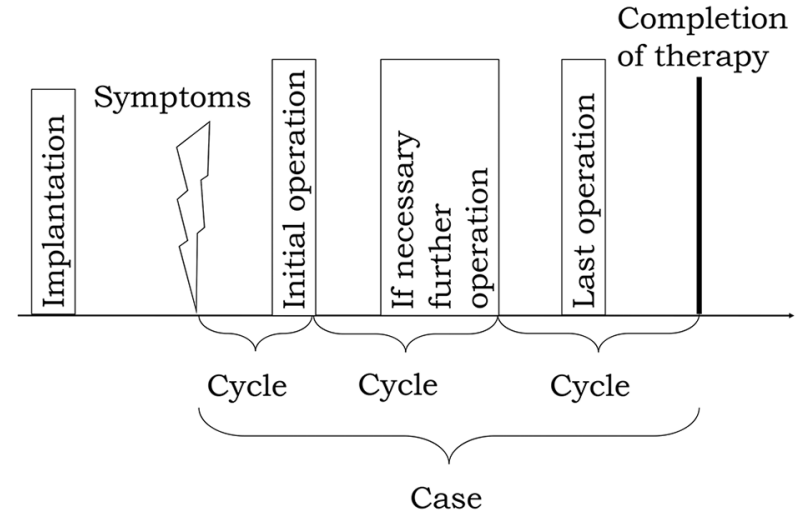

Figure 2. Case definition. The first cycle was from beginning of symptoms to the end of the first operation. Then, the next cycle starts and ends with the next operation. The last cycle ends with the termination of the case including the postoperative phase. All cycles together are one case.

Statistical analysis was performed with GraphPad Prism 5 (GraphPad Software Inc., La Jolla, USA) and Vassarstats (www.vassarstats.com). Statistical significance was considered for a $p$-value less than $0.05(p \leq 0.05)$, or if the $95 \%$ confidence intervals (CI) had no overlapping. Furthermore, sensitivity, specificity, positive (ppv) and negative predictive value (npv) as well as area under the curve (AUC) was determined as ROC curve with GraphPad Prism.

\section{Results}

General data. A total of 183 patients were included. Of these, there were $95(51.9 \%)$ female and $88(48.1 \%)$ male patients. In $\mathrm{n}=108(59.0 \%)$ patients there was an infection of the hip, in $\mathrm{n}=73(39.9 \%)$ patients an infection of the knee and in $\mathrm{n}=2(1.1 \%)$ patients an infection of both hip and knee. The mean age was $66.6(12.8-93.4)$ years. Fifty patients (27.3\%) were included for more than one case, which leads to $1.44(\mathrm{~min} / \mathrm{max} 1-7)$ included cases per patient. Table I shows the associated risk factors and outcomes.

Cycles and cases. Overall, 505 synovial aspiration cultures could be included. Regarding the above-mentioned case definition, this leads to 264 cases. There were 126 (47.7\%) female and $138(52.3 \%)$ male patients. In $\mathrm{n}=165(62.5 \%)$ cases there was an infection of the hip, in $\mathrm{n}=99(37.5 \%)$ cases an infection of the knee and in $n=134(50.8 \%)$ cases there had been a prior revision. The mean number of prior revisions was $\mathrm{n}=3.58(\mathrm{~min} / \mathrm{max} 1-28)$. In $\mathrm{n}=104(39.4 \%)$ we found an early infection, in $\mathrm{n}=112(42.4 \%)$ a delayed infection and in $\mathrm{n}=48$ $(18.2 \%)$ of the cases a late infection.

Diagnostic value of synovial culture aspiration. Table II shows the results for synovial cultures overall and itemized for hip and knee. In comparison to the hip culture the 
Table I. Associated risk factors for periprosthetic infection (PJI) and outcome of all patients.

\begin{tabular}{lc}
\hline Risk factors for PJI & $\mathrm{n}(\%)$ \\
\hline Adiposity & $66(36.1 \%)$ \\
Immunosuppression & $39(21.3 \%)$ \\
Diabetes & $28(15.3 \%)$ \\
Smoking & $25(13.7 \%)$ \\
Rheumatoid arthritis & $21(11.5 \%)$ \\
Prior PJI on same joint & $18(9.8 \%)$ \\
Malignant tumour on same joint & $17(9.3 \%)$ \\
Prior septic arthritis on same joint & $12(6.6 \%)$ \\
Anaemia before implant of arthroplasty & $22(12.0 \%)$ \\
No anaemia before implant of arthroplasty & $36(19.7 \%)$ \\
None of these risc factors & $37(20.2 \%)$ \\
\hline Outcome & $\mathrm{n}(\%)$ \\
\hline Discharge with arthroplasty & $177(67.0 \%)$ \\
No infection present in follow-up & $110(41.7 \%)$ \\
Discharge without infection, but no follow-up & $44(16.7 \%)$ \\
Relapse of infection & $94(35.6 \%)$ \\
Permanent arthrodesis & $21(8.0 \%)$ \\
Permanent Girdlestone & $27(10.2 \%)$ \\
Amputation & $29(11.0 \%)$ \\
Death & $16(6.1 \%)$ \\
\hline
\end{tabular}

PJI: Periprosthetic joint infection.

sensitivity for the knee culture was significantly higher, as well as the NPV and the AUC $(p=0.038)$.

Diagnostic value of synovial culture aspiration in view of time point of infection. In Table III the value of synovial culture for different time points of PJI are presented. We were unable to find any significant differences.

Diagnostic value of synovial culture aspiration in view of antibiotic treatment. There were no significantly different values for synovial culture aspiration with or without (at least two weeks without antibiotics) ongoing antibiotic treatment. Table IV shows the results.

Diagnostic value of synovial culture aspiration in view of prosthesis removal. In case of complete removal of prosthesis, the sensitivity and AUC are significantly reduced, whereas the specificity was comparable in all three different situations with prosthesis in situ, partial removal or complete removal of prosthesis (Table V and Figure 3). The PPV was reduced if the prosthesis was completely removed, however, it was not significant due to a broad range of the CI.

\section{Discussion}

We demonstrated, that the diagnostic value of synovial culture varies within the sometimes complex treatment of
Table II. Results of all synovial aspiration cultures and the subgroup of hip and knee cultures.

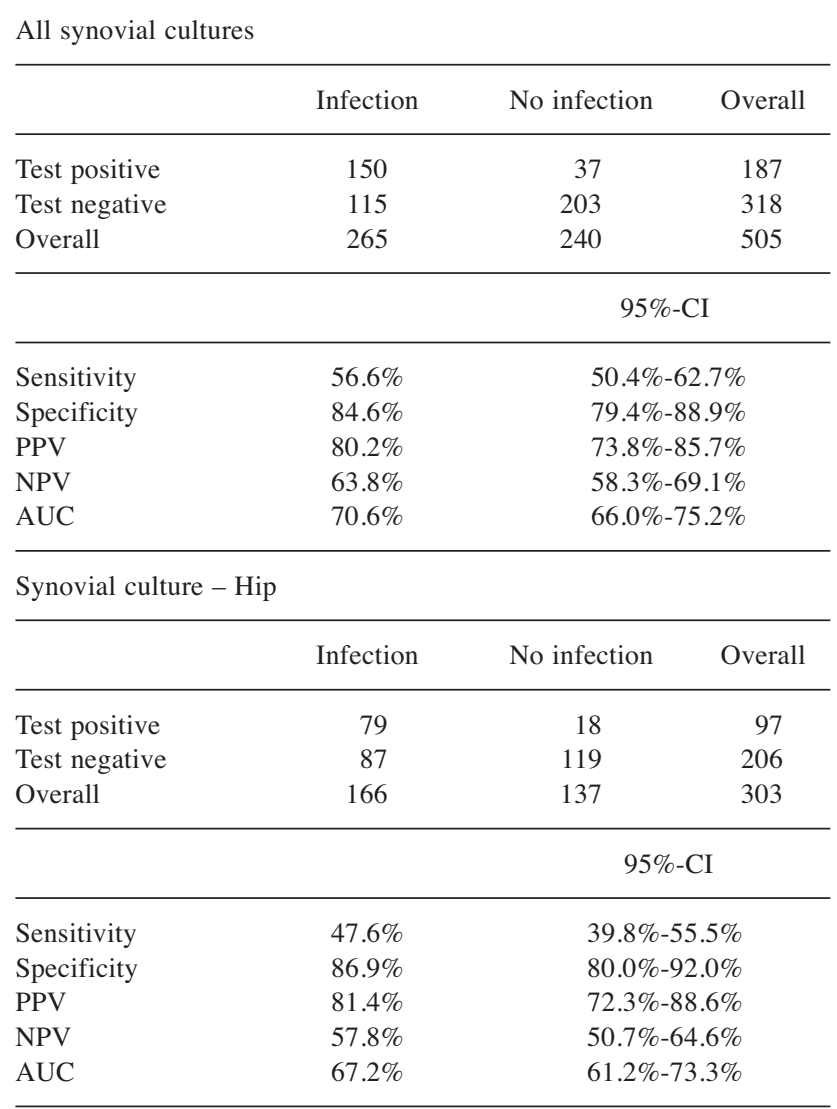

Synovial culture - Knee

\begin{tabular}{lccr}
\hline & Infection & No infection & Overall \\
\hline Test positive & 71 & 19 & 90 \\
Test negative & 28 & 84 & 112 \\
Overall & 99 & 103 & 202 \\
\hline \multicolumn{2}{c}{$95 \%$-CI } \\
\hline Sensitivity & \multicolumn{2}{c}{$61.8 \%-80.3 \%$} \\
Specificity & $71.7 \%$ & $72.7 \%-88.5 \%$ \\
PPV & $81.6 \%$ & $69.0 \%-86.8 \%$ \\
NPV & $78.9 \%$ & $65.9 \%-82.7 \%$ \\
AUC & $75.0 \%$ & $69.9 \%-83.4 \%$ \\
\hline
\end{tabular}

PPV: Positive predictive value; NPV: negative predictive value; AUC: area under the curve; CI: confidence interval.

these infections depending on treatment stage. Furthermore, due to the low sensitivity in the case of complete removal, an aspiration before reimplantation does not seem to make sense in order to reliably exclude infection.

According to the Renner criteria, in addition to the detection of a fistula, the joint puncture aspiration is the 
Table III. Results of all synovial aspiration cultures in the subgroup of early, delayed and late infections.

Synovial culture - Early infections

\begin{tabular}{lccc}
\hline & Infection & No infection & Overall \\
\hline Test positive & 42 & 10 & 52 \\
Test negative & 41 & 58 & 99 \\
Overall & 83 & 68 & 151 \\
\hline & & \multicolumn{2}{c}{$95 \%$-CI } \\
\hline Sensitivity & $50.6 \%$ & $39.4 \%-61.8 \%$ \\
Specificity & $85.3 \%$ & $74.6 \%-92.7 \%$ \\
PPV & $80.8 \%$ & $67.5 \%-90.4 \%$ \\
NPV & $58.6 \%$ & $48.2 \%-68.4 \%$ \\
AUC & $68.0 \%$ & $59.4 \%-76.5 \%$ \\
\hline
\end{tabular}

Synovial culture - Delayed infections

\begin{tabular}{lccr}
\hline & Infection & No infection & Overall \\
\hline Test positive & 80 & 11 & 91 \\
Test negative & 50 & 95 & 145 \\
Overall & 130 & 106 & 236 \\
\hline \multicolumn{2}{c}{$95 \%-\mathrm{CI}$} \\
\hline Sensitivity & \multicolumn{2}{|c}{$52.6 \%-69.9 \%$} \\
Specificity & $61.5 \%$ & $82.2 \%-94.7 \%$ \\
PPV & $89.6 \%$ & $79.4 \%-93.8 \%$ \\
NPV & $87.9 \%$ & $57.2 \%-73.2 \%$ \\
AUC & $65.5 \%$ & $69.3 \%-81.8 \%$ \\
\hline
\end{tabular}

Synovial culture - Late infections

\begin{tabular}{lccc}
\hline & Infection & No infection & Overall \\
\hline Test positive & 28 & 16 & 44 \\
Test negative & 24 & 50 & 74 \\
Overall & 52 & 66 & 118 \\
\hline & & \multicolumn{2}{c}{$95 \%$-CI } \\
\hline Sensitivity & $53.9 \%$ & $39.5 \%-67.8 \%$ \\
Specificity & $75.8 \%$ & $63.6 \%-85.5 \%$ \\
PPV & $63.6 \%$ & $47.8 \%-77.6 \%$ \\
NPV & $67.6 \%$ & $55.7 \%-78.0 \%$ \\
AUC & $64.8 \%$ & $54.7 \%-75.0 \%$ \\
\hline
\end{tabular}

PPV: Positive predictive value; NPV: negative predictive value; AUC: area under the curve; $\mathrm{CI}$ : confidence interval.

essential possibility to detect a periprosthetic joint infection preoperatively (7). Different methods are used to analyse the joint aspiration puncture obtained. Diagnosis usually begins with light microscopy of a gram-stained direct specimen. This method delivers very quick results, is highly specific, but has only moderate sensitivity $(12,13)$.
Table IV. Results of all synovial aspiration cultures dependence of actual antibiotic treatment.

Synovial culture - With actual antibiotic treatment

\begin{tabular}{lccc}
\hline & Infection & No infection & Overall \\
\hline Test positive & 85 & 20 & 105 \\
Test negative & 60 & 96 & 156 \\
Overall & 145 & 116 & 261 \\
\hline & \multicolumn{2}{c}{$95 \%$-CI } \\
\hline Sensitivity & $58.6 \%$ & $50.2 \%-66.7 \%$ \\
Specificity & $82.8 \%$ & $74.6 \%-89.1 \%$ \\
PPV & $81.0 \%$ & $72.1 \%-88.0 \%$ \\
NPV & $61.5 \%$ & $53.4 \%-69.2 \%$ \\
AUC & $70.7 \%$ & $64.4 \%-77.0 \%$ \\
\hline
\end{tabular}

Synovial culture - Without actual antibiotic treatment

\begin{tabular}{lccr}
\hline & Infection & No infection & Overall \\
\hline Test positive & 65 & 17 & 82 \\
Test negative & 55 & 107 & 162 \\
Overall & 120 & 124 & 244 \\
\hline & & \multicolumn{2}{c}{$95 \%-\mathrm{CI}$} \\
\hline Sensitivity & $54.2 \%$ & $44.8 \%-63.3 \%$ \\
Specificity & $86.3 \%$ & $79.0 \%-91.8 \%$ \\
PPV & $79.3 \%$ & $68.9 \%-87.4 \%$ \\
NPV & $66.1 \%$ & $58.2 \%-73.3 \%$ \\
AUC & $70.2 \%$ & $63.6 \%-76.9 \%$ \\
\hline
\end{tabular}

PPV: Positive predictive value; NPV: negative predictive value; AUC: area under the curve; CI: confidence interval.

The routine method for examining joint aspiration punctures in the diagnosis of periprosthetic infections is cultural incubation in a nutrient broth. However, this takes a lot of time and is therefore unsuitable for acute diagnosis, but can then be helpful in further therapy by creating an antibiogram $(11,14)$. The sensitivity of the cultural analysis of joint puncture aspiration in the primary diagnosis of periprosthetic infections fluctuates in the literature; a detailed list of the results of other studies can be found in Table VI.

In comparison to the hip culture the sensitivity for the knee culture was significantly higher, as well as the NPV and the AUC ( $p=0.038$ ). One possible cause for these findings could be the procedure of joint aspiration. As the knee joint is relatively superficial, the puncture is, in contrast to the hip joint, usually performed without sonography and/or x-ray control. But even with these imaging procedures aspiration of seroma without connection to the hip joint or punctio sicca is not unlikely $(15,16)$. 
Table V. Results of all synovial aspiration cultures with prosthesis in situ, partial removal of the prosthesis and complete removal of the prosthesis.

Synovial culture aspiration - Prosthesis in situ

\begin{tabular}{lccc}
\hline & Infection & No infection & Overall \\
\hline Test positive & 121 & 24 & 145 \\
Test negative & 68 & 100 & 168 \\
Overall & 189 & 124 & 313 \\
\hline \multicolumn{2}{c}{$95 \%$-CI } \\
\hline Sensitivity & \multicolumn{2}{|c}{$56.7 \%-70.9 \%$} \\
Specificity & $64.0 \%$ & $72.6 \%-87.2 \%$ \\
PPV & $80.7 \%$ & $76.4 \%-89.1 \%$ \\
NPV & $83.5 \%$ & $51.7 \%-67.0 \%$ \\
AUC & $59.5 \%$ & $66.6 \%-78.1 \%$ \\
\hline
\end{tabular}

Synovial culture aspiration - Partial removal of prosthesis

\begin{tabular}{lccc}
\hline & Infection & No infection & Overall \\
\hline Test positive & 21 & 4 & 25 \\
Test negative & 10 & 23 & 33 \\
Overall & 31 & 27 & 58 \\
\hline \multicolumn{2}{c}{$95 \%$-CI } \\
\hline Sensitivity & \multicolumn{2}{|c}{$48.6 \%-83.3 \%$} \\
Specificity & $67.7 \%$ & $66.3 \%-95.8 \%$ \\
PPV & $85.2 \%$ & $63.9 \%-95.5 \%$ \\
NPV & $84.0 \%$ & $51.3 \%-84.4 \%$ \\
AUC & $69.7 \%$ & $63.8 \%-89.1 \%$ \\
\hline
\end{tabular}

Synovial culture aspiration - Complete removal of prosthesis

\begin{tabular}{lccc}
\hline & Infection & No infection & Overall \\
\hline Test positive & 8 & 9 & 17 \\
Test negative & 37 & 80 & 117 \\
Overall & 45 & 89 & 134 \\
\hline & \multicolumn{2}{c}{$95 \%$-CI } \\
\hline Sensitivity & \multicolumn{2}{|c}{$8.0 \%-32.1 \%$} \\
Specificity & $17.8 \%$ & $81.7 \%-95.3 \%$ \\
PPV & $89.9 \%$ & $23.0 \%-72.3 \%$ \\
NPV & $47.1 \%$ & $59.1 \%-76.7 \%$ \\
AUC & $68.4 \%$ & $43.3 \%-64.4 \%$ \\
\hline
\end{tabular}

PPV: Positive predictive value; NPV: negative predictive value; AUC: area under the curve; CI: confidence interval.

Regarding our results of the hip culture in comparison with the existing literature sensitivity and specificity are in the range of other studies (sensitivity: $47.6 \%$ vs. $58.2 \%$; specificity: $86.9 \%$ vs. $78 \%$; see also Table VI for comparison) (17-21). A similar pattern can be seen for the synovial cultures of the knee, at least for sensitivity. Eight

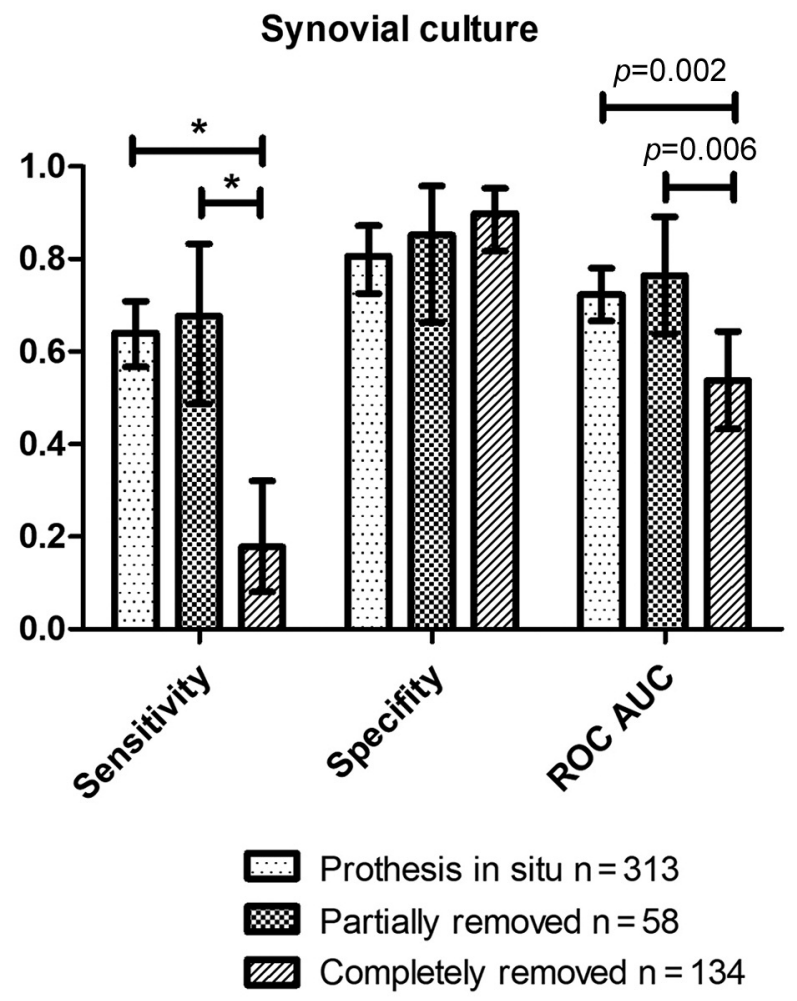

Figure 3. Results for synovial culture aspiration with prosthesis in situ, partial removal and complete removal with presentation of the statistically significant differences (*significant with respect to $95 \%$ CI, p-value for ROC in the graphic).

studies exclusively examined the value of synovial cultures from knee joint aspiration at the start of therapy (22-29). The mean sensitivity was $70 \%$, the mean specificity $97 \%$ (our study: $71.7 \%$ and $81.6 \%$ ) (Table VI). The lower specificity for knee synovial culture of our study is noticeable. Nonetheless, it should be mentioned that in contrast to other studies, samples that were obtained within the sometimes complex therapeutic regimen were also taken into account in our study. This could explain the difference in this issue. We were unable to find any significant differences regarding the timing of the infection, although the general distribution of our data is comparable to the literature $(30,31)$.

Furthermore, there were no significantly different values in our study for synovial culture with or ongoing antibiotic treatment. The number of existing studies for the diagnostic value of synovial cultures in dependence of antibiotic treatment is sparse and contradictory though. Mendelez et al. found a higher sensitivity for synovial culture in patients receiving antibiotic treatment in the last 30 days (29). However, the authors forego a precise analysis of the duration of the antibiotic pause. In contrast, Barrack and colleagues showed a lower sensitivity, if the samples were 
in vivo 35 : $2409-2416(2021)$

Table VI. Diagnostic value of synovial culture with prosthesis in the literature in comparison to this study.

Other studies regarding diagnostic value of synovial culture with prosthesis

\begin{tabular}{|c|c|c|c|c|}
\hline Author, year & Investigation & Pat. $(n=)$ & Sens. & Spec. \\
\hline Morgenstern et al., 2018 (34) & Hip and Knee & 142 & $52 \%$ & $98 \%$ \\
\hline Prieto-Borja et al., 2018 (30) & Hip, Knee, Shoulder & 200 & $54.8 \%$ & $94.2 \%$ \\
\hline Janz et al., 2016 (17) & Hip & 69 & $68 \%$ & $50 \%$ \\
\hline Melendez et al., 2016 (29) & Knee & 284 & $76.1 \%$ & $97.4 \%$ \\
\hline Melendez et al., 2016 (29) & Knee with antibiotic therapy within 30 days & 70 & $85.4 \%$ & \\
\hline Janz et al., 2015 (28) & Knee & 109 & $67 \%$ & $97 \%$ \\
\hline Ryu et al., 2014 (27) & Knee & 89 & $72 \%$ & $96 \%$ \\
\hline Cazanave et al., 2013 (35) & Hip and Knee & 434 & $66.3 \%$ & $96.9 \%$ \\
\hline Janz et al., 2013 (36) & Hip and Knee & 59 & $91 \%$ & $81 \%$ \\
\hline Janz et al., 2013 (18) & Hip & 102 & $66 \%$ & $96 \%$ \\
\hline Gomez et al., 2012 (37) & Hip and Knee & 366 & $64.7 \%$ & $96.9 \%$ \\
\hline Tohtz et al., 2010 (20) & Hip & 64 & $15.8 \%$ & $97.8 \%$ \\
\hline Müller et al., 2009 (19) & Hip & 106 & $61 \%$ & $50 \%$ \\
\hline Fink et al., 2008 (24) & Knee & 145 & $72.5 \%$ & $95.2 \%$ \\
\hline Gallo et al., 2008 (31) & Hip, Knee and Elbow & 115 & $43.5 \%$ & $93.8 \%$ \\
\hline Fuerst et al., 2005 (25) & Knee & 75 & $68.8 \%$ & $96.6 \%$ \\
\hline Williams et al., 2004 (21) & Hip & 273 & $80 \%$ & $94 \%$ \\
\hline Kordelle et al., 2004 (26) & Knee & 45 & $50 \%$ & $100 \%$ \\
\hline Virolainen et al., 2002 (12) & Hip and Knee & 68 & $75 \%$ & $100 \%$ \\
\hline Teller et al., 2000 (38) & All arthroplasties & 166 & $28 \%$ & $99 \%$ \\
\hline Barrack et al, 1997 (22) & Knee & 67 & $55 \%$ & $96 \%$ \\
\hline Barrack et al., 1997 (22) & Knee without prior antibiotic therapy & 53 & $75 \%$ & $96 \%$ \\
\hline Duff et al., 1996 (23) & Knee & 43 & $100 \%$ & $100 \%$ \\
\hline Glithero et al, 1993 (39) & Hip and Knee & 54 & $89 \%$ & $97 \%$ \\
\hline Levitsky et al., 1991 (40) & Hip and Knee & 70 & $67 \%$ & $96 \%$ \\
\hline Johnson et al., 1988 (41) & Hip, Knee and Shoulder & 28 & $12 \%$ & $81 \%$ \\
\hline This study & Hip and Knee & 183 & $57 \%$ & $86 \%$ \\
\hline
\end{tabular}

Pat.: Patients; N: numbers; Sens.: sensitivity; Spec.: specificity.

taken under antibiotic therapy (22). Unfortunately, our study has limited suitability for a differentiated statement of the effect of antibiotic therapy. Due to the retrospective design, it remains in some cases unclear, whether an antibiogram adapted therapy was already carried out and which exact therapy was performed in the ambulatory sector.

In this study, we found that the sensitivity and AUC significantly reduced in case of complete removal of the prosthesis. This underlines the importance of the biofilm on the implant surface for the pathogenesis and diagnosis of periprosthetic infections. On the one hand, the removal of the biofilm is essential for the treatment of a foreign body-associated infection (7). As our data show, however, the diagnostic value of synovial cultures decreases enormously after removal of the implant and consequently the biofilm. These results support that the probability of a successful cultural pathogen detection in the puncture depends crucially on the release of planktonic germs from the biofilm on the implant surface, as already described by other authors $(15,32)$. Moreover, it seems, that it does not make sense to collect synovial samples for cultural analysis in the prosthesis-free interval to exclude a persistent infection.

The specificity was comparable in all three different situations with prosthesis in situ, partial removal or complete removal of prosthesis. However, one has to be careful with interpretation of the high specificity of $89.9 \%$ in complete removal, as this means a false positive rate of $10.1 \%$ and thus could lead to an unnecessary additional operation. On the other hand, as the specificity of partial removal, for example in megaprosthesis after trauma or tumour is comparable with the complete removal, the synovial culture could be a decision support especially in these critical patients.

The study had certain imitations and strengths. First, it should be pointed out that using a different definition system of periprosthetic infection might have achieved different results. We opted for the consensus classification, but some authors use different definitions, which reduces comparability (33). Further, as this is a retrospective study, a standardization of the puncture/aspiration itself (although these standards did not change in our clinic during the study) cannot be guaranteed with absolute certainty. As mentioned above, in 
contrast to other studies we included samples within the complex regime of periprosthetic infections as well, which limits the comparability to them. On the other hand, we consider our results to be valid precisely because this comes closer to the clinical course than an isolated observation of the synovial aspiration culture at the beginning of therapy.

\section{Conclusion}

It could be shown that the sensitivity decreases significantly when the prosthesis material is completely removed, whereas the results with partially removed prosthesis material are comparable with the results with the prosthesis material left in place. Due to the low sensitivity, obtaining several synovial cultures in the prosthesis-free interval to exclude persistence of infection does not seem reasonable.

\section{Conflicts of Interest}

The Authors declare no conflicts of interest.

\section{Author's Contributions}

CM: Study design and realization, analysing and data interpretation, manuscript writing. SL: Study design and realization, data collection, analysing and data interpretation, manuscript reviewing. TG: Data collection, analysis and data interpretation, manuscript reviewing. MOE: Data collection, analysis and data interpretation, manuscript reviewing. TOP: Analysis and data interpretation, manuscript reviewing. TS: analysis and data interpretation, manuscript reviewing. CK: Reviewing of the manuscript. MO: Study design and realization, analysis and data interpretation, manuscript reviewing

\section{Acknowledgements}

The data of this study are part of the doctoral thesis of SL.

\section{References}

1 Abad CL and Haleem A: Prosthetic joint infections: an update. Curr Infect Dis Rep 20(7): 15, 2018. PMID: 29789958. DOI: 10.1007/s11908-018-0622-0

2 Otto-lambertz C, Yagdiran A, Wallscheid F, Eysel P and Jung N: Periprosthetic infection in joint replacement. Dtsch Arztebl Int 114(20): 347-353, 2017. PMID: 28610654. DOI: 10.3238/ arztebl.2017.0347

3 Renz N, Müller M, Perka C and Trampuz A: [Implant-associated infections - Diagnostics]. Chirurg 87(10): 813-821, 2016. PMID: 27439064. DOI: 10.1007/s00104-016-0234-x

4 Bozic KJ and Ries MD: The impact of infection after total hip arthroplasty on hospital and surgeon resource utilization. J Bone Joint Surg Am 87(8): 1746-1751, 2005. PMID: 16085614. DOI: 10.2106/JBJS.D.02937

5 Zimmerli W, Trampuz A and Ochsner PE: Prosthetic-joint infections. N Engl J Med 351(16): 1645-1654, 2004. PMID: 15483283. DOI: 10.1056/NEJMra040181
6 Trampuz A and Zimmerli W: Diagnosis and treatment of implant-associated septic arthritis and osteomyelitis. Curr Infect Dis Rep 10(5): 394-403, 2008. PMID: 18687204. DOI: 10.1007/ s11908-008-0064-1

7 Renner L, Perka C, Trampuz A and Renz N: [Treatment of periprosthetic infections]. Chirurg 87(10): 831-838, 2016. PMID: 27484827. DOI: $10.1007 / \mathrm{s} 00104-016-0255-5$

8 Frommelt L: [Aspiration of joint fluid for detection of the pathogen in periprosthetic infection]. Orthopade 37(10): 102734; quiz 1035-6, 2008. PMID: 18797842. DOI: 10.1007/s00132008-1345-y

9 Morawietz L, Gehrke T, Classen RA, Barden B, Otto M, Hansen T, Aigner T, Stiehl P, Neidel J, Schröder JH, Frommelt L, Schubert T, Meyer-Scholten C, König A, Ströbel P, Rader ChP, Kirschner S, Lintner F, Rüther W, Skwara A, Bos I, Kriegsmann $\mathrm{J}$ and Krenn V: [Proposal for the classification of the periprosthetic membrane from loosened hip and knee endoprostheses]. Pathologe 25(5): 375-384, 2004. PMID: 15257415. DOI: 10.1007/s00292-004-0710-9

10 Morawietz L, Classen RA, Schröder JH, Dynybil C, Perka C, Skwara A, Neidel J, Gehrke T, Frommelt L, Hansen T, Otto M, Barden B, Aigner T, Stiehl P, Schubert T, Meyer-Scholten C, König A, Ströbel P, Rader CP, Kirschner S, Lintner F, Rüther W, Bos I, Hendrich C, Kriegsmann J and Krenn V: Proposal for a histopathological consensus classification of the periprosthetic interface membrane. J Clin Pathol 59(6): 591-597, 2006. PMID: 16731601. DOI: $10.1136 /$ jcp.2005.027458

11 Zimmerli W: Infection and musculoskeletal conditions: Prosthetic-joint-associated infections. Best Pract Res Clin Rheumatol 20(6): 1045-1063, 2006. PMID: 17127196. DOI: 10.1016/j.berh.2006.08.003

12 Virolainen P, Lähteenmäki H, Hiltunen A, Sipola E, Meurman O and Nelimarkka O: The reliability of diagnosis of infection during revision arthroplasties. Scand J Surg 91(2): 178-181, 2002. PMID: 12164519. DOI: 10.1177/145749690209100208

13 Wouthuyzen-Bakker M, Shohat N, Sebillotte M, Arvieux C, Parvizi $J$ and Soriano A: Is Gram staining still useful in prosthetic joint infections? J Bone Jt Infect 4(2): 56-59, 2019. PMID: 31011508. DOI: 10.7150/jbji.31312

14 Hughes HC, Newnham R, Athanasou N, Atkins BL, Bejon P and Bowler IC: Microbiological diagnosis of prosthetic joint infections: a prospective evaluation of four bacterial culture media in the routine laboratory. Clin Microbiol Infect 17(10): 1528-1530, 2011. PMID: 21851478. DOI: 10.1111/j.14690691.2011.03597.x

15 Winkler T, Trampuz A, Hardt S, Janz V, Kleber C and Perka C: [Periprosthetic infection after hip arthroplasty]. Orthopade 43(1): 70-78, 2014. PMID: 24414232. DOI: 10.1007/s00132-013-2132-y

16 Perka C and Haas N: [Periprosthetic infection]. Chirurg 82(3): 218-226, 2011. PMID: 21340589. DOI: 10.1007/s00104-0102014-3

17 Janz V, Bartek B, Wassilew GI, Stuhlert M, Perka CF and Winkler T: Validation of synovial aspiration in girdlestone hips for detection of infection persistence in patients undergoing 2stage revision total hip arthroplasty. J Arthroplasty 31(3): 684687, 2016. PMID: 26521130. DOI: 10.1016/j.arth.2015.09.053

18 Janz V, Wassilew GI, Hasart O, Tohtz S and Perka C: Improvement in the detection rate of PJI in total hip arthroplasty through multiple sonicate fluid cultures. J Orthop Res 31(12): 2021-2024, 2013. PMID: 23893822. DOI: 10.1002/jor.22451 
19 Müller M, Morawietz L, Hasart O, Strube P, Perka C and Tohtz S: [Histopathological diagnosis of periprosthetic joint infection following total hip arthroplasty : use of a standardized classification system of the periprosthetic interface membrane]. Orthopade 38(11): 1087-1096, 2009. PMID: 19690832. DOI: 10.1007/s00132-009-1471-1

20 Tohtz SW, Müller M, Morawietz L, Winkler T and Perka C: Validity of frozen sections for analysis of periprosthetic loosening membranes. Clin Orthop Relat Res 468(3): 762-768, 2010. PMID: 19768513. DOI: 10.1007/s11999-009-1102-5

21 Williams JL, Norman P and Stockley I: The value of hip aspiration versus tissue biopsy in diagnosing infection before exchange hip arthroplasty surgery. J Arthroplasty 19(5): 582586, 2004. PMID: 15284978. DOI: 10.1016/j.arth.2003.11.011

22 Barrack RL, Jennings RW, Wolfe MW and Bertot AJ: The Coventry Award. The value of preoperative aspiration before total knee revision. Clin Orthop Relat Res (345): 8-16, 1997. PMID: 9418615.

23 Duff GP, Lachiewicz PF and Kelley SS: Aspiration of the knee joint before revision arthroplasty. Clin Orthop Relat Res (331): 132-139, 1996. PMID: 8895629. DOI: 10.1097/00003086199610000-00018

24 Fink B, Makowiak C, Fuerst M, Berger I, Schäfer P and Frommelt L: The value of synovial biopsy, joint aspiration and C-reactive protein in the diagnosis of late peri-prosthetic infection of total knee replacements. J Bone Joint Surg Br 90(7): 874-878, 2008. PMID: 18591595. DOI: 10.1302/0301620X.90B7.20417

25 Fuerst M, Fink B and Rüther W: [The value of preoperative knee aspiration and arthroscopic biopsy in revision total knee arthroplasty]. Z Orthop Ihre Grenzgeb 143(1): 36-41, 2005. PMID: 15754230 . DOI: $10.1055 / \mathrm{s}-2004-836252$

26 Kordelle J, Klett R, Stahl U, Hossain H, Schleicher I and Haas $\mathrm{H}$ : [Infection diagnosis after knee-TEP-implantation]. Z Orthop Ihre Grenzgeb 142(3): 337-343, 2004. PMID: 15250008. DOI: $10.1055 / \mathrm{s}-2004-818772$

27 Ryu SY, Greenwood-Quaintance KE, Hanssen AD, Mandrekar JN and Patel R: Low sensitivity of periprosthetic tissue PCR for prosthetic knee infection diagnosis. Diagn Microbiol Infect Dis 79(4): 448-453, 2014. PMID: 24972853. DOI: 10.1016/ j.diagmicrobio.2014.03.021

28 Janz V, Wassilew GI, Kribus M, Trampuz A and Perka C: Improved identification of polymicrobial infection in total knee arthroplasty through sonicate fluid cultures. Arch Orthop Trauma Surg 135(10): 1453-1457, 2015. PMID: 26350385. DOI: $10.1007 / \mathrm{s} 00402-015-2317-4$

29 Melendez DP, Greenwood-Quaintance KE, Berbari EF, Osmon DR, Mandrekar JN, Hanssen AD and Patel R: Evaluation of a Genus- and Group-Specific Rapid PCR assay panel on synovial fluid for diagnosis of prosthetic knee infection. J Clin Microbiol 54(1): 120-126, 2016. PMID: 26537446. DOI: 10.1128/ JCM.02302-15

30 Prieto-Borja L, Auñón Á, Blanco A, Fernández-Roblas R, Gadea I, García-Cañete J, Parrón R and Esteban J: Evaluation of the use of sonication of retrieved implants for the diagnosis of prosthetic joint infection in a routine setting. Eur J Clin Microbiol Infect Dis 37(4): 715-722, 2018. PMID: 29270861. DOI: $10.1007 / \mathrm{s} 10096-017-3164-8$

31 Gallo J, Kolar M, Dendis M, Loveckova Y, Sauer P, Zapletalova $\mathrm{J}$ and Koukalova D: Culture and PCR analysis of joint fluid in the diagnosis of prosthetic joint infection. New Microbiol 31(1): 97-104, 2008. PMID: 18437847

32 Mariconda M, Ascione T, Balato G, Rotondo R, Smeraglia F, Costa GG and Conte M: Sonication of antibiotic-loaded cement spacers in a two-stage revision protocol for infected joint arthroplasty. BMC Musculoskelet Disord 14: 193, 2013. PMID: 24192225. DOI: $10.1186 / 1471-2474-14-193$

33 Parvizi J, Jacovides C, Zmistowski B and Jung KA: Definition of periprosthetic joint infection: is there a consensus? Clin Orthop Relat Res 469(11): 3022-3030, 2011. PMID: 21751038. DOI: $10.1007 / \mathrm{s} 11999-011-1971-2$

34 Morgenstern C, Cabric S, Perka C, Trampuz A and Renz N: Synovial fluid multiplex PCR is superior to culture for detection of low-virulent pathogens causing periprosthetic joint infection. Diagn Microbiol Infect Dis 90(2): 115-119, 2018. PMID: 29191466. DOI: 10.1016/j.diagmicrobio.2017.10.016

35 Cazanave C, Greenwood-Quaintance KE, Hanssen AD, Karau MJ, Schmidt SM, Gomez Urena EO, Mandrekar JN, Osmon DR, Lough LE, Pritt BS, Steckelberg JM and Patel R: Rapid molecular microbiologic diagnosis of prosthetic joint infection. J Clin Microbiol 51(7): 2280-2287, 2013. PMID: 23658273. DOI: $10.1128 / \mathrm{JCM} .00335-13$

36 Janz V, Wassilew GI, Hasart O, Matziolis G, Tohtz S and Perka $\mathrm{C}$ : Evaluation of sonicate fluid cultures in comparison to histological analysis of the periprosthetic membrane for the detection of periprosthetic joint infection. Int Orthop 37(5): 931936, 2013. PMID: 23525549. DOI: 10.1007/s00264-013-1853-1

37 Gomez E, Cazanave C, Cunningham SA, GreenwoodQuaintance KE, Steckelberg JM, Uhl JR, Hanssen AD, Karau MJ, Schmidt SM, Osmon DR, Berbari EF, Mandrekar J and Patel R: Prosthetic joint infection diagnosis using broad-range PCR of biofilms dislodged from knee and hip arthroplasty surfaces using sonication. J Clin Microbiol 50(11): 3501-3508, 2012. PMID: 22895042. DOI: 10.1128/JCM.00834-12

38 Teller RE, Christie MJ, Martin W, Nance EP and Haas DW: Sequential indium-labeled leukocyte and bone scans to diagnose prosthetic joint infection. Clin Orthop Relat Res (373): 241-247, 2000. PMID: 10810483. DOI: 10.1097/00003086-20000400000029

39 Glithero PR, Grigoris P, Harding LK, Hesslewood SR and McMinn DJ: White cell scans and infected joint replacements. Failure to detect chronic infection. J Bone Joint Surg Br 75(3): 371-374, 1993. PMID: 8496202. DOI: 10.1302/0301620X.75B3.8496202

40 Levitsky KA, Hozack WJ, Balderston RA, Rothman RH, Gluckman SJ, Maslack MM and Booth RE Jr: Evaluation of the painful prosthetic joint. Relative value of bone scan, sedimentation rate, and joint aspiration. J Arthroplasty 6(3): 237-244, 1991. PMID: 1940929. DOI: 10.1016/s0883-5403(06)80170-1

41 Johnson JA, Christie MJ, Sandler MP, Parks PF Jr, Homra L and Kaye JJ: Detection of occult infection following total joint arthroplasty using sequential technetium-99m HDP bone scintigraphy and indium-111 WBC imaging. J Nucl Med 29(8): 1347-1353, 1988. PMID: 3404252. 